\title{
Head-Eye movement control tests in patients with chronic neck pain; Inter-observer reliability and discriminative validity
}

Eveline Della Casa ${ }^{1,2+}$, Jutta Affolter Helbling ${ }^{2,3^{*}}$, André Meichtry ${ }^{2}$, Hannu Luomajoki ${ }^{2}$ and Jan Kool ${ }^{2}$

\begin{abstract}
Background: Head-eye movement control deficit is an identified problem in patients with chronic neck pain, particularly in cases of whiplash associated disorders (WAD). To date, there is no evidence concerning the reliability and validity of visually assessed active head-eye movement control tests. Therefore, the objectives of the present cross-sectional study were, a) to develop a test battery; and b) to investigate inter-observer reliability and discriminative validity in patients with chronic neck pain compared to healthy controls.
\end{abstract}

Methods: The study was conducted at two physiotherapy clinics in Switzerland. Ethics Committee approval was obtained. Ten active head-eye coordination tests, on 23 patients with chronic neck pain and associated symptoms and 19 healthy controls, were videotaped. The tests included eye movements in the neutral head position and $45^{\circ}$ relative neck rotation, gaze stability and sequential head-eye movements. All tests were performed in the sitting and standing positions. Two blinded physiotherapists independently rated the randomized videos. Performance was rated as "negative", "moderately positive" or "clearly positive". Weighted kappa (wK) and 95\% confidence intervals (Cl) were calculated to investigate inter-observer reliability. Good reliability was defined as wK $>0.5$ with a lower boundary of $95 \% \mathrm{Cl}>0.2$. Odds ratios (to define cut-off points) and the distribution of the classificator, numbers of positive tests, were calculated.

Results: Three out of ten tests showed "excellent" (wK 0.82 to 0.86), five out of ten tests showed "substantial" (wK 0.69 to 0.79) and two out of ten tests showed "moderate" (wK 0.54 to 0.59 ) reliability. Results were comparable in the sitting and standing positions. On average, three out of five tests were rated positive in patients and one out of five tests was rated positive in healthy controls. An odds ratio of 13.3 to 18.6 was obtained using $\geq 2 / 5$ tests as a cut-off point.

Conclusion: Visual assessment by physiotherapists of head-eye movement control tests is reliable. The test battery is able to discriminate between patients with chronic neck pain and healthy controls. There were no differences in performance between the sitting and standing positions. The test battery can therefore be reduced to five tests. Further research is needed to identify the test-retest stability and responsiveness.

Keywords: Head-eye movement control, Chronic neck pain, Whiplash associated disorders, Reliability, Discriminative validity, Assessment

\footnotetext{
* Correspondence: jutta.affolter@gamt.ch

${ }^{\dagger}$ Equal contributors

${ }^{2}$ Zurich University of Applied Sciences, School of Health Professions, Institute

of Physiotherapy, 8400, Winterthur, Switzerland

${ }^{3}$ Provital Physiotherapy\& GRAVITYtraining, Büelstrasse 29, 8132, Egg,

Switzerland

Full list of author information is available at the end of the article
}

\section{Biomed Central}

(c) 2014 Della Casa et al.; licensee BioMed Central Ltd. This is an open access article distributed under the terms of the Creative Commons Attribution License (http://creativecommons.org/licenses/by/2.0), which permits unrestricted use, distribution, and reproduction in any medium, provided the original work is properly cited. 


\section{Background}

Neck pain is a common problem, with lifetime prevalence in the general population worldwide of up to $71 \%[1,2]$. Impaired head-eye movement control is an identified problem in patients with chronic neck pain, particularly in patients with whiplash associated disorders (WAD). Symptoms associated with head-eye movement control impairment are dizziness, headache, light-headedness and visual disorders [3]. Movements requiring the coordination of neck, head and eyes are known to provoke those symptoms. A well-known example is driving a car after whiplash trauma, where Gimse found changed eye movements during car driving [4]. He also found altered eye movements during reading and differences in smooth pursuit eye movements with the head in the neutral position [5]. In patient anamnesis, symptom provocation was found during walking, fast head rotations and observation of moving objects. Oculomotor dysfunctions after cervical trauma were described by Hildingssons and colleagues [6-8]. Yahia and colleagues found abnormal dynamic and static balance in patients with chronic neck pain and dizziness [9]. Humphreys and colleagues [10] investigated neck pain in 180 patients: one third reported dizziness.

Following Treleaven and colleagues [3], dizziness is a frequent symptom in patients with neck pain and WAD. They assumed the underlying mechanism to be a convergence dysfunction of the sensorimotor input from the cervical spine, the vestibulum and the visual postural system. This theory is also supported by other authors [11-14]. Heikkilä et al. [11], found a reduced range of cervical motion and decreased upper cervical proprioception, which affects the voluntary eye movement. Peterson [13] postulated the head-neck motor system as an ideal model for understanding issues of complex motor control and Armstrong [14], mentioned that sensory information from neck proprioceptors is processed in tandem with information from the vestibulum, cerebellum and cortex.

Following diagnosis of a dysfunction of convergence, the management of an intervention is based on the clinical presentation and the functional impairment. The following assessments are recommended to measure the functional impairment and are used in clinical practice: joint position error (JPE) [12], "The Fly" movement method [15], smooth pursuit neck torsion test (SPNT) [16], postural stability and balance, as well as oculomotory and head-eye coordination tests $[17,18]$.

In this study, the selection of our tests was influenced by some of the research tests outlined above. The test battery had to meet the following criteria: have the ability to transfer the research results into practice; be simple; performed without high-tech equipment; easy to learn; and, preferably, be low cost. The test battery of five tests was performed in the sitting position and the standing position. The latter, with feet together to achieve a more challenging balance position [19].

Eye movement was tested according to Jull and colleagues [19]. They tested eye-follow in both neutral and in relative $45^{\circ}$ neck rotation, using the elements of the smooth pursuit neck torsion test from Tjell [16]. Tjell compared eye movements performed with the head in neutral position to a position in $45^{\circ}$ relative head rotation. He reported significant differences between these two positions in patients with whiplash trauma compared to patients with vestibular pathology. Because of the unavailablity of equipment for standardizing eye movement velocity, in the present study the eye movement tests were performed differently to Tjell et al. [16]. The participants were asked to move their eyes horizontally from side to side as quickly as possible, while actively maintaining their initial fixed head position.

Treleaven and colleagues [3] showed that patients with WAD have decreased velocity of movement in the sequential head and eye movement test and in the gaze stability task, compared to healthy controls. Furthermore, patients with WAD have a reduced active range of motion of the cervical spine in the gaze stability test. These altered movement patterns were associated with a higher score in the Neck Disability Index (NDI) [3]. However, this data was collected using sophisticated laboratory equipment to measure eye movement in association with head movement. A physiotherapeutic diagnosis, is based on visual observation of the patients' impaired head-eye movement. According to Treleaven [20] visual clinical observation of the quality of head and eye movement, in conjunction with symptom provocation, is the suggested method for assessment of oculomotory dysfunction. Qualitative and quantitative aspects of movement, as well as symptom provocation, are references for physiotherapeutic intervention $[10,19]$. Range of motion and velocity of movement is difficult to measure without technical equipment but the quality of movement control can be visually inferred [21]. To date, the reliability of visual observation of head-eye coordination tests has not been investigated.

The main objectives of the present study were to:

a) Develop a test battery

b) Investigate the inter-tester reliability of the visual observation of ten videotaped head-eye coordination tests

c) Determine the discriminative validity of these tests in patients with chronic neck pain compared to healthy controls

Our hypothesis was that, "Visual assessments by physiotherapists of head-eye movement control deficit are reliable and that these tests are able to discriminate between controls and patients". 
We also hypothesized a greater head-eye control deficit in the standing position compared to sitting, since the latter is a more challenging balance position and requires increased coordination.

\section{Methods \\ Design}

A cross-sectional study, conforming to the Declaration of Helsinki, was performed at two physiotherapy outpatient clinics in Switzerland. Ethical approval was obtained from the Ethics Committee of Canton Zurich. Written informed consent was provided by all participating subjects, as well as for permission to use the photographs in the manuscript. Ten head-eye movement control tests were videotaped from a face-front perspective, with five performed in the sitting and five in the standing position [19]. Two experienced (OMT IFOMPT qualified) blinded physiotherapists independently rated the randomized videos.

\section{Participants}

Based on a previous study by Treleaven et al. [3], a sample size of 40 participants was used in this study. All patients were recruited over a 2-month period and had been referred to the clinics either by a general practitioner, chiropractor or other physiotherapist. During the same period, healthy controls were recruited from a training centre and from our circle of acquaintances.

Males and females aged between 25 and 70 years were included (Table 1). Patients were required to have suffered from non-specific chronic neck pain during the last 3 months, an NDI score of $>10 / 100[22,23]$ and a minimum of one symptom associated with head-eye movement control impairment, such as dizziness, lightheadedness or a visual disorder.

The NDI is a reliable and valid instrument in the assessment of the limiting factors in patients with chronic neck pain [22-24]. (Scoring interpretation for the NDI [23]: $<10 / 100=$ none, $10-28 / 100=$ mild, $30-48 / 100=$ moderate, $50-68 / 100=$ severe, $>70 / 100=$ complete).

For inclusion, patients had to have a minimum of $45^{\circ}$ active range of motion rotation to both sides and be able to stand freely with feet together without risk of falling.

Table 1 Characteristics of participants

\begin{tabular}{llll}
\hline Group & Patients & Controls & Total \\
\hline Number & 23 & 19 & 42 \\
Female/Male & $23 / 0$ & $12 / 7$ & $35 / 7$ \\
Age: mean / min.-max. & $50.7 / 29-70$ years & $50.9 / 31-70$ years & \\
NDI mean / min.-max. & $32.2 / 10-62$ & $3.3 / 0-8$ & \\
Neck Trauma yes / no & $17 / 6$ & $2 / 17$ & \\
\hline
\end{tabular}

-Neck disability index score NDI 0-100 points.

-Age $(p$-value $)=0.939$.
Included in the study were 17 patients with neck trauma and $6(26 \%)$ without trauma.

Excluded from the study were subjects with known eye diseases, specifically blindness, strabismus, nystagmus, trochlear nerve injury, central nervous system disease and vestibular disease (such as Menière's disease). Furthermore, a history of ear surgery, dizziness caused by vascular problems and neck pain caused by cervical radiculopathy, were grounds for exclusion.

Healthy controls were excluded if they had reported visual disturbances, dizziness or neck pain during the last six months. Two subjects in the control group reported minor historical neck trauma, but had not experienced any symptoms for many years.

An age-matched control group was recruited because there is evidence to show that age affects head-eye coordination $[25,26]$.

\section{Standardized testing procedure}

The order of the tests was standardized:

1) Eye movements neutral; 2) Gaze stability; 3) Sequential head and eye movements; 4) Eye movements in $45^{\circ}$ relative neck rotation to the right; and 5) Eye movements in $45^{\circ}$ relative neck rotation to the left. These five tests were performed in the sitting position and then repeated in the standing with feet together position. The markers and camera were placed at participants' eye level. The participants were given verbal instructions and were asked to move as precisely and as quickly as possible during the tests. Each test was recorded on video for 10 seconds. No resting time was allowed between tests, except to allow a short instruction time for the following test. In the case of symptom provocation, subjects were advised to stop. Spectacles and contact lenses were worn as usual.

\section{Test facility}

To prevent patient falls, the test facility (Figures 1 and 2) was situated in a corner of the room. An equilateral triangle, with sides of $1 \mathrm{~m}$ in length, was marked on the floor. A chair and a footprint were positioned at one side of the triangle and two sticks in styrofoam sockets at the other sides. Both sticks had coloured markers whitch could be adjusted to participants' eye level. The camera (type: Canon Legria HF R205) was positioned on a tripod exactly in the centre of the triangle and could also be adjusted to eye level. The angle between the camera and one side of the triangle amounted to $30^{\circ}$, resulting in a standardized gaze change of $30^{\circ}$ to both sides.

Three arrows were marked on the chair for the start of the sitting tests and three on the footprint for the standing tests. Position 1 was placed exactly in front of the camera and marked the neutral head position. Position 2 was placed at an angle of $45^{\circ}$ to the right of the camera 


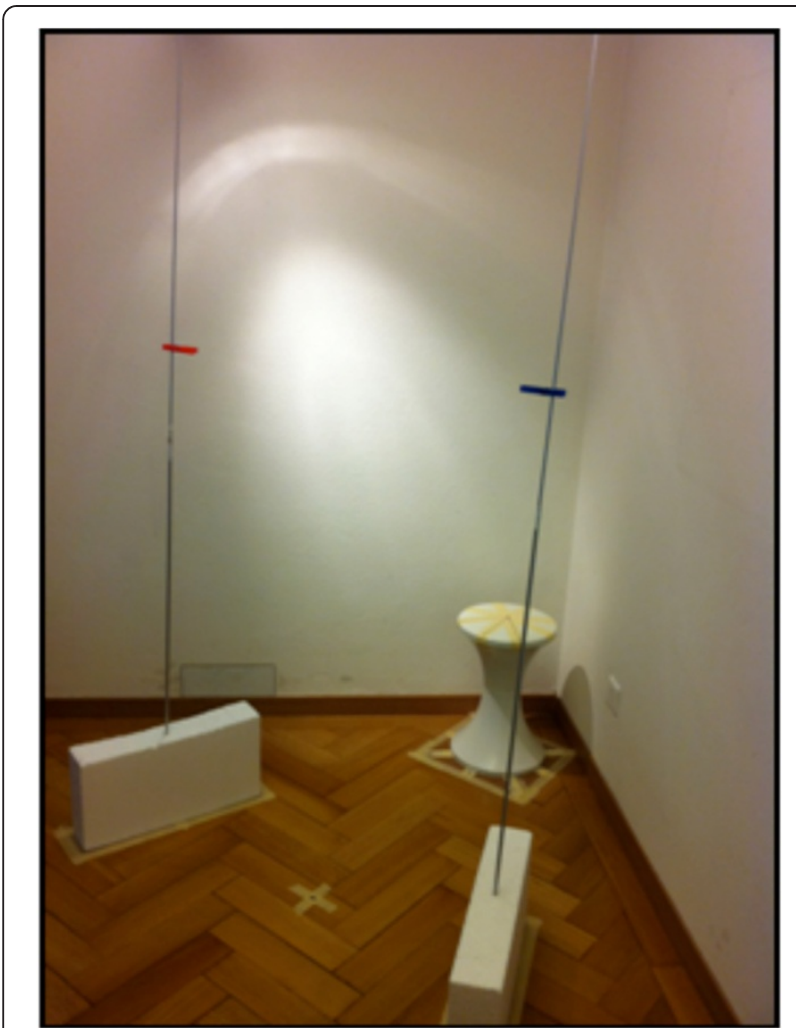

Figure 1 Test facility for sitting posture. Blue and red markers are at participants' eye level. The cross on the floor marks the camera position (also at eye level).

(Position 1) and Position 3 was placed $45^{\circ}$ to the left of the camera. For the tests in $45^{\circ}$ relative neck rotation to the right, the participants sat or stood on position 3 and turned their head towards the camera, thus creating the $45^{\circ}$ relative neck rotation to the right.

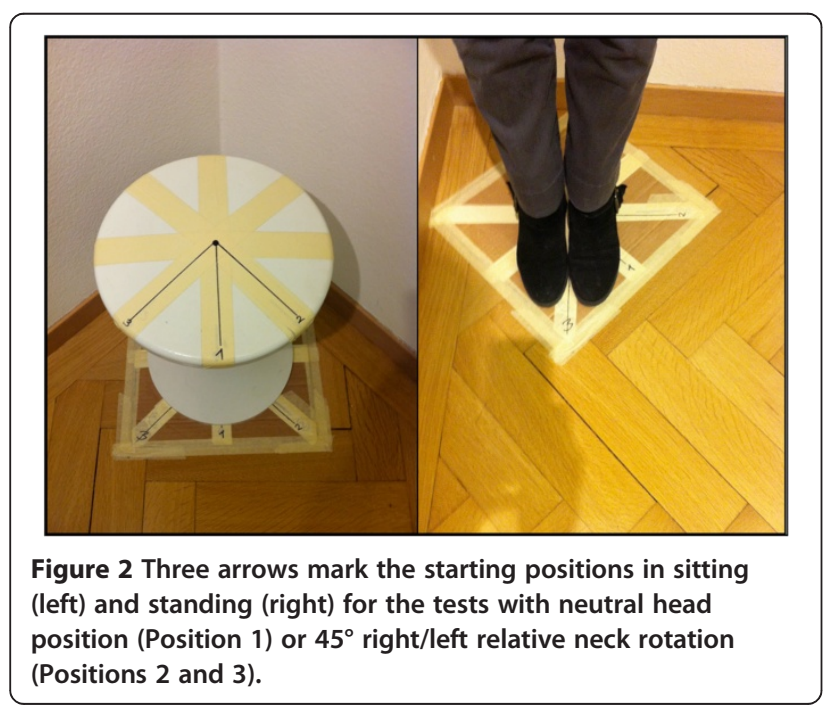

\section{Tests}

\section{Eye movements}

Eye movements (Figure 3) were performed according to Jull and colleagues [19].

\section{Procedure}

Test in the sitting position: participants sat on the chair with an upright posture at position 1 . The markers were adjusted to eye level.

Test in the standing position: The participants stood on the footprint with feet together at position 1 . The markers were adjusted to eye level.

The subjects were required to maintain their head in the neutral position while moving their eyes sideways from one marker to the other as fast as possible for 10 seconds.

Instruction: "Keep your head in the previously adjusted neutral position while moving your eyes between the two markers as fast as possible".

The test rating scale is shown in Table 2.

\section{Gaze stability}

The test is shown in Figure 4 and the rating scale in Table 3.

\section{Procedure}

Test in the sitting position: participants sat on the chair with an upright posture at Position 1. The camera was adjusted to eye level.

Test in the standing position: participants stood on the footprint with feet together at Position 1. The camera was adjusted to eye level.

The subjects were asked to hold a stable gaze on the camera while moving their head to left and to right as far and as fast as possible for 10 seconds.

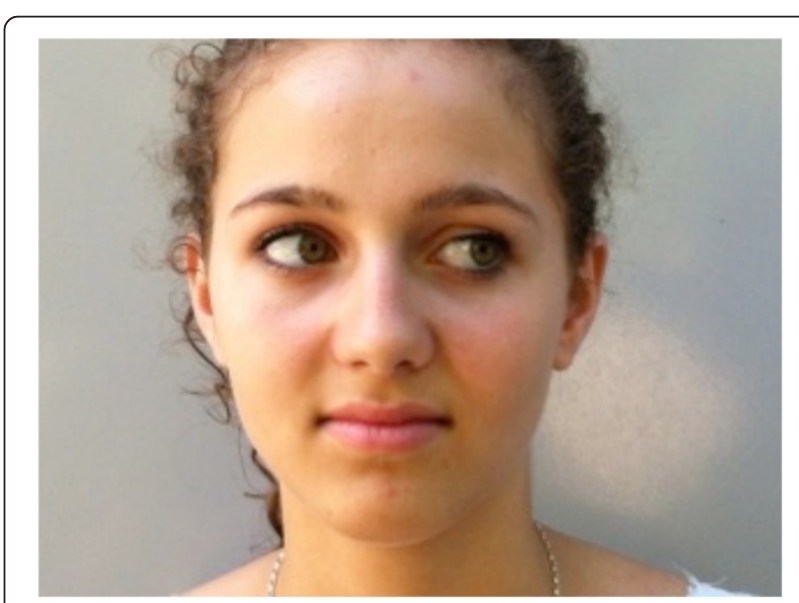

Figure 3 Eye movements to right and left while maintaining the head in the neutral position. 
Table 2 Rating scale of eye movements in neutral head position

\begin{tabular}{ll}
\hline Rating & Definition \\
\hline $0=$ negative & $\begin{array}{l}\text { Smooth, precise, fast eye movements, } \\
\text { fast change of gaze direction, head } \\
\text { remains stable }\end{array}$ \\
$1=$ moderately positive & $\begin{array}{l}\text { Slightly irregular eye movements. Short } \\
\text { stops before changing gaze direction. } \\
\text { Head slightly unstable }\end{array}$ \\
& $\begin{array}{l}\text { Eye movements clearly slower or irregular. } \\
\text { Prolonged maintenance of gaze direction } \\
\text { before changing direction. Obvious head } \\
\text { movement. Test cannot be performed }\end{array}$ \\
\hline
\end{tabular}

Instruction: "Hold your gaze stable on the camera while moving your head left and right as far and as fast as possible".

\section{Sequential head and eye movements}

Dissociated sequential head and eye movements according to Treleaven and colleagues [3] were performed in a range of motion of $30^{\circ}$ to both sides.

\section{Procedure}

Test in the sitting position: participants sat on the chair with upright posture at Position 1 . The markers were adjusted to eye level.

Test in the standing position: participants stood on the footprint with feet together on position 1. The markers were adjusted to eye level.

Starting in the neutral position, the participants were to move their eyes to the right marker with the head remaining still. Subsequently, they were to rotate the head in the same direction with gaze remaining fixed. With the head facing the right marker, the eyes were then to focus on the left marker, followed by the leftwards rotation of the head with fixed gaze. These movements were to be repeated for 10 seconds.

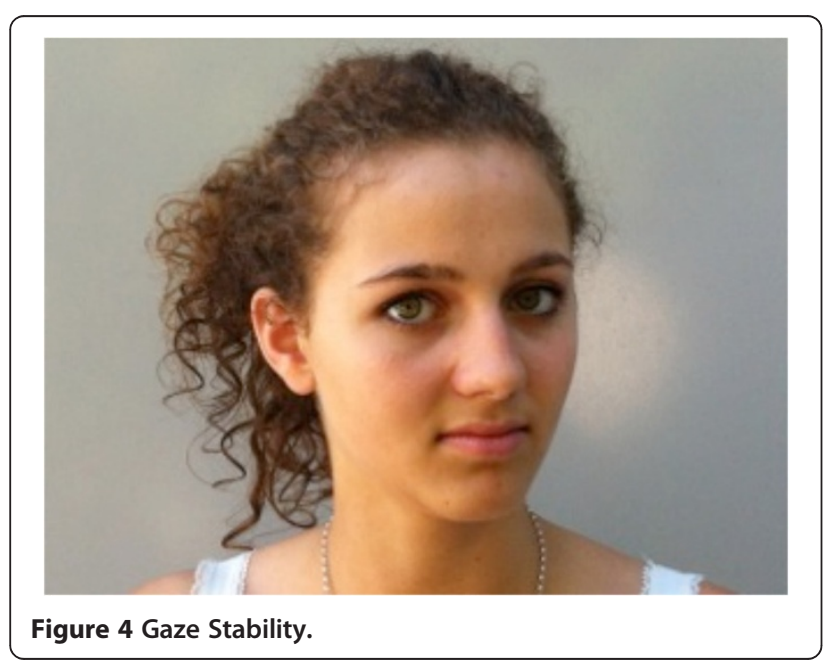

Table 3 Rating scale of gaze stability

\begin{tabular}{ll}
\hline Rating & Definition \\
\hline $0=$ negative & $\begin{array}{l}\text { Gaze stable. Smooth, well-coordinated, } \\
\text { precise and fast movement of the head, } \\
\text { fluent change of head movement direction }\end{array}$ \\
$1=$ moderate positive & $\begin{array}{l}\text { Gaze stable. Slightly irregular head } \\
\text { movement }\end{array}$ \\
$2=$ strong positive & $\begin{array}{l}\text { Gaze repeatedly unstable. Head movement } \\
\text { slow and irregular }\end{array}$ \\
\hline
\end{tabular}

Instruction: "Move your eyes to the right marker while holding your head still. Then, rotate your head to the same marker, keeping your gaze fixed. Thereafter, move your eyes to the left marker while keeping your head on the right side, then rotate your head to the left marker. Do this as precisely and as fast as possible. Continue for 10 seconds" (Figure 5).

\section{Eye movements with $45^{\circ}$ relative neck rotation to right/left}

The eye movements test, as previously described, was repeated with $45^{\circ}$ relative neck rotation to the left and right in the sitting and standing positions.

\section{Procedure}

Test in the sitting position: participants sat on the chair at Position 2 for the left rotation and at Position 3 for the right rotation. They were then asked to turn the head towards the camera, thus creating the $45^{\circ}$ relative neck rotation. The markers were adjusted to eye level.

Test in the standing position: participants stood on the footprint with feet together at Position 2 or 3 . They were then asked to turn the head towards the camera, thus creating the $45^{\circ}$ relative neck rotation. The markers were adjusted to eye level.

Participants were to hold this position and move the gaze between the right and the left markers as fast as possible for 10 seconds.

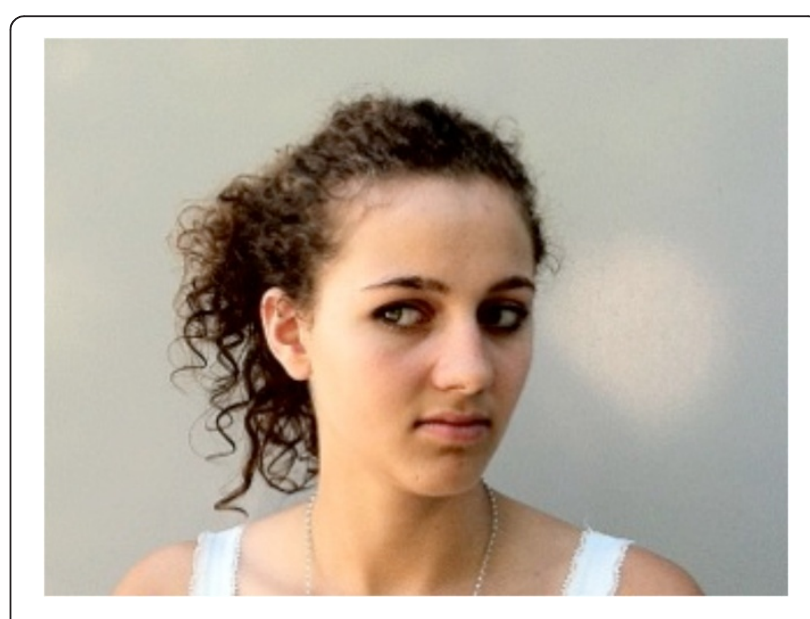

Figure 5 Sequential head and eye movements. 
Instruction: "Move your eyes between the two markers as fast as possible, while maintaining your neck in the fixed position". (Figure 6)

The test rating scale was the same as in the previous test for eye movements in the neutral position (Table 2).

Detailed description of the test protocol is available in Additional file 1 .

\section{Test video rating}

Randomization: The videos were randomized. Each participant was allocated a number for the whole test battery. These numbers were mixed in a basket and drawn out by an author. Through this method, the order of the videos for the rating process was prearranged.

Two experienced physiotherapists (OMT IFOMPT qualified), blinded to all participants' data, independently rated the randomized videos. They had had no prior specific training, apart from a few minutes of an instructionvideo. Raters were allowed to view the videos more than once, if necessary. Each test was rated following the criteria described above (Tables 2, 3 and 4).

\section{Statistical analysis}

Software package $\mathrm{R}$ was used for statistical analysis. A weighted Cohen's kappa (wK) coefficient and confidence interval [95\%] was calculated for each test. According to Landis, $w K>0.80$ was defined as almost perfect, $0.60 \leq \mathrm{wK}<0.80$ as substantial, $0.40 \leq \mathrm{wK}<0.60$ as moderate, $0.21 \leq \mathrm{wK}<0.40$ as fair and $\mathrm{wK}<0.20$ as slight agreement [27]. In this study, acceptable reliability was defined as $\mathrm{wK}>0.5$ and the lower lever of the confidence interval $>0.2$. In addition to $w K$ the percentage agreement was determined.

The distribution of positive and negative results was calculated for each test. To determine discriminative validity,

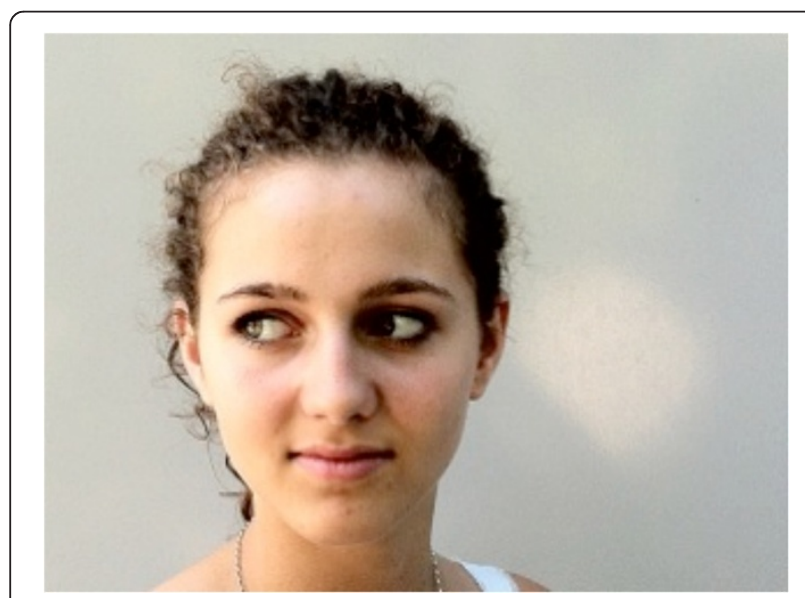

Figure 6 Eye movements with $45^{\circ}$ relative neck rotation to the right.
Table 4 Rating scale of sequential head and eye movements

\begin{tabular}{ll}
\hline Rating & Definition \\
\hline $0=$ negative & $\begin{array}{l}\text { Clear, regular, smooth, dissociated } \\
\text { movements of head and eyes. }\end{array}$ \\
$1=$ moderately positive & $\begin{array}{l}\text { Slightly decelerated eye movements, } \\
\text { occasional associated eye-head movements, } \\
\text { head unstable. }\end{array}$ \\
& $\begin{array}{l}\text { Clearly decelerated, irregular and often } \\
\text { associated eye-head movements, test not } \\
\text { feasible. }\end{array}$ \\
\hline
\end{tabular}

the number of positive tests (moderate or strong positive) was used as a classifier.

To quantify the performance of the classification, for different cut-off values of the number of positive tests, we calculated the following absolute frequencies: true positives (TP), true negatives (TN), false positives (FP) and false negatives (FN). From these quantities, sensitivity $(\mathrm{Sn})$, specificity $(\mathrm{Sp})$, positive predictive value (PPV) and negative predictive value (NPV) were calculated.

Since a reference test for head-eye movement control impairment is not available, Diagnostic Odds Ratios (DOR) for the different cut-off values were calculated, which defined the associations between ratings and group affiliation. The DOR is the factor by which the chance of impairment is increased with a positive compared to a negative test result. As a global criterion, the Receiver Operating Characteristic (ROC) curve with the Area under the Curve (AUC) was computed. In our context, the AUC is equivalent to the probability that the number of positive tests is larger for impaired than for healthy subjects.

\section{Results}

Subjects

Patient characteristics (gender, age, NDI score) are described in Table 1. There was no significant difference in age between patients and healthy controls ( $p$-value 0.939).

Included patients had an NDI score of 10 to 62/100, which implies an inhomogeneous representation of symptoms.

\section{Inter-observer reliability}

The results of the inter-observer reliability analysis (weighted kappa coefficient and 95\% confidence interval) are presented in Table 5 . The weighted kappa coefficient was almost perfect $(w K>0.8)$ in three tests, substantial (wK $0.69-0.79$ ) in five tests and moderate in two tests (wK 0.54 and 0.59 ). The $95 \%$ confidence interval was $>0.2$ in all tests $(0.29$ to 0.97$)$. Excellent inter-tester reliability was found for gaze stability and sequential head and eye movements in sitting position, and eye movements in $45^{\circ}$ relative neck rotation to the right side in standing position. 
Table 5 Results inter-observer reliability: weighted kappa (wK) 95\% confidence interval $(95 \% \mathrm{CI})$, percentage agreement (\% Agreement)

\begin{tabular}{|c|c|c|c|}
\hline Tests: Sitting position & wK & $95 \% \mathrm{Cl}$ & $\%$ Agreement \\
\hline Eye movements & 0.72 & $0.55-0.88$ & 73 \\
\hline Gaze stability & 0.86 & $0.75-0.97$ & 85 \\
\hline Sequential head and eye movement & 0.86 & $0.76-0.97$ & 83 \\
\hline Eye movements in $45^{\circ}$ relative neck rotation to the right & 0.54 & $0.29-0.79$ & 69 \\
\hline Eye movements in $45^{\circ}$ relative neck rotation to the left & 0.79 & $0.62-0.97$ & 85 \\
\hline Tests: Standing position & wK & $95 \% \mathrm{Cl}$ & $\%$ Agreement \\
\hline Eye movements & 0.78 & $0.61-0.95$ & 85 \\
\hline Gaze stability & 0.59 & $0.36-0.82$ & 66 \\
\hline Sequential head and eye movement & 0.69 & $0.53-0.85$ & 61 \\
\hline Eye movements in $45^{\circ}$ relative neck rotation to the right & 0.82 & $0.67-0.96$ & 85 \\
\hline Eye movements in $45^{\circ}$ relative neck rotation to the left & 0.70 & $0.50-0.90$ & 80 \\
\hline
\end{tabular}

\section{Discriminative validity}

On average, three out of five tests in sitting and in standing positions were positive for participants with chronic neck pain. On average, only one test was positive for healthy controls. Results were comparable in the sitting and standing positions.
Figures 7 and 8 display the distribution of the numbers of positive tests for the tests in sitting and standing. There was no significant difference between the two positions, or between raters.

The receiver operating characteristic (ROC) curves with the area under curve AUC $(95 \% \mathrm{CI})$ show a distribution of
Patients

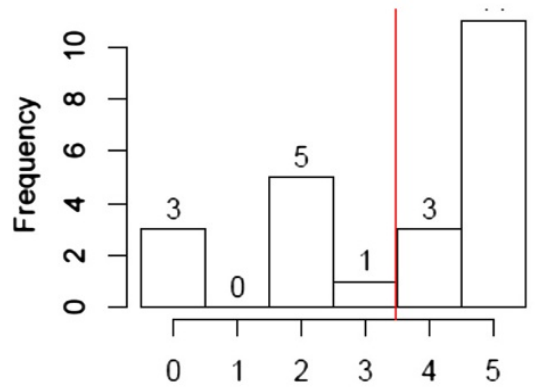

Sitting (number of positive tests), rater 1

\section{Patients}

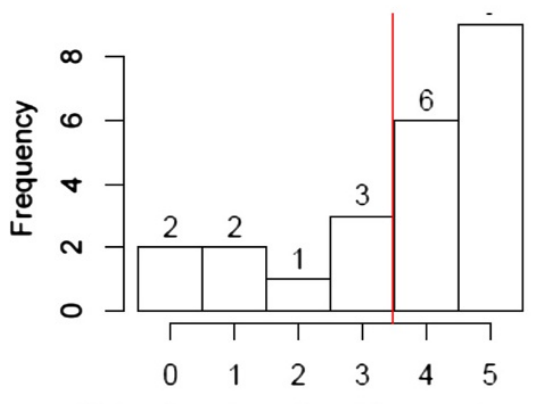

Sitting (number of positive tests), rater 2

\section{Controls}

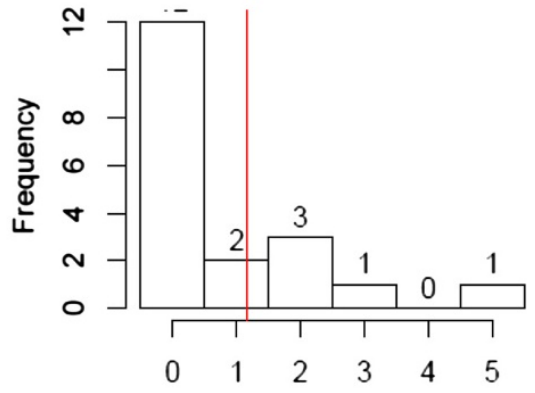

Sitting (number of positive tests), rater 1

\section{Controls}

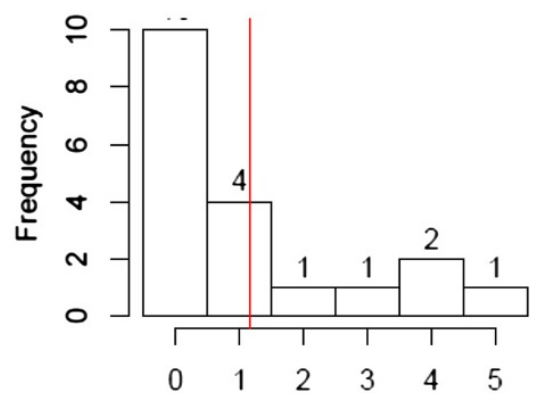

Sitting (number of positive tests), rater 2

Figure 7 Distribution of positive tests in sitting. The red line displays the mean number of positive tests. Results were comparable for Rater 1 and Rater 2 


\section{Patients}

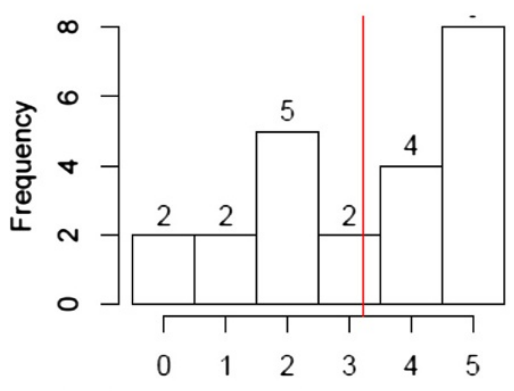

Standing (number of positive tests), rater 1

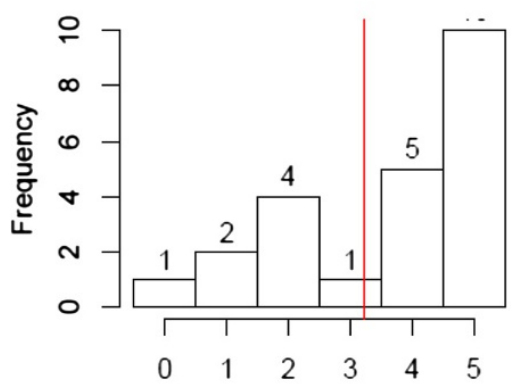

Standing (number of positive tests), rater 2

\section{Controls}

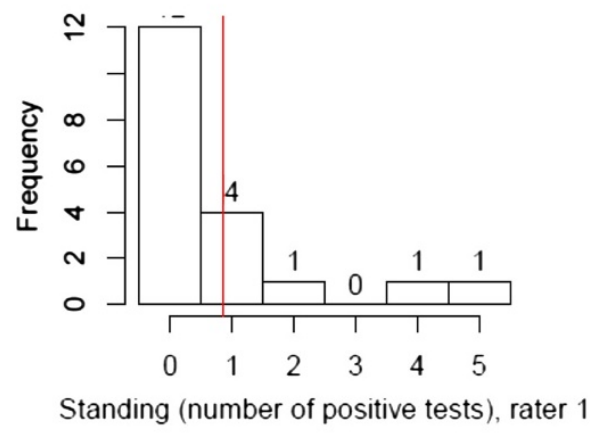

Controls

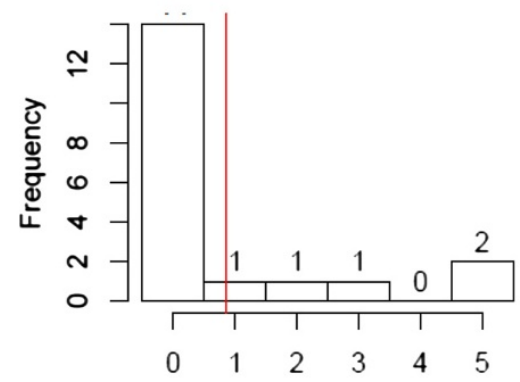

Standing (number of positive tests), rater 2

Figure 8 Distribution of positive tests in standing. The red line displays the mean number of positive tests. Results were comparable for Rater 1 and Rater 2.

the numbers of positive tests in sitting (Figure 9) of 83-85\% and in standing (Figure 10) of $85-87 \%$

The Diagnostic Odds Ratio (DOR), test in sitting, of Rater 1 and Rater 2 are 18.6 and 13.3 respectively, using 2 positive tests as the cut-off point.

For the clinical diagnosis of head-eye movement control impairment, a cut-off point of 2 positive ratings out of 5 is recommended. (Tables 6 and 7)

\section{Discussion}

Visual rating by physiotherapists of head-eye movement control tests is reliable. Predefined criteria for inter-tester reliability were fulfilled. Similar results for inter-tester reliability were reported by Luomajoki et al. [21], when videos of movement control tests of the lumbar spine were visually evaluated.

In the present study, the discriminative validity was supported with DOR values above 13 to 18 , for persons with 2 or more positive tests out of 5 . This means that patients with chronic neck pain and associated symptoms, such as dizziness, visual disorders, etc., are up to 13-18 times more likely to have 2 or more tests positive than healthy controls. For the clinical diagnosis of head-eye movement control impairment, a cut-off point of 2 positive results out of 5 tests in sitting or standing position is recommended.
Results showed there was no significant difference between the numbers of positive tests in the sitting and standing positions. Therefore, the hypothesis of an observable increase in head-eye movement control impairment in the standing with feet together position was not confirmed. Since all participants were first tested in sitting and then in standing position, the results also imply that there was no relevant accumulation of fatigue or learning adaptation. The test battery can, therefore, be reduced to five tests. We recommend the tests to be performed in the sitting position in order to minimise the risk of falling in patients with dizziness.

Visible differences between controls and patients were identified in the tests performed: patients with chronic neck pain showed a decelerated velocity of head and eye movements and distinctive movement quality. These results are in line with previous research [3].

The strength of these tests is that they can be performed by all subjects and do not require technical equipment, such as an oculograph. Results showed that quality differences were visually identifiable during the10 seconds performance per test. This is important for general clinical use, since assessment is fast and requires little installation. These tests could be of assistance in the daily clinical practice of physiotherapists, in 


\section{Test in sitting, rater 1}

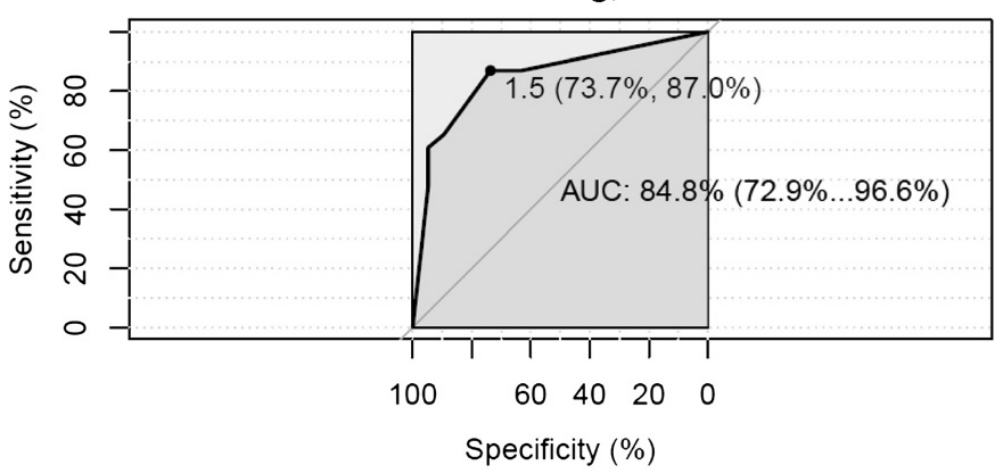

Test in sitting, rater 2

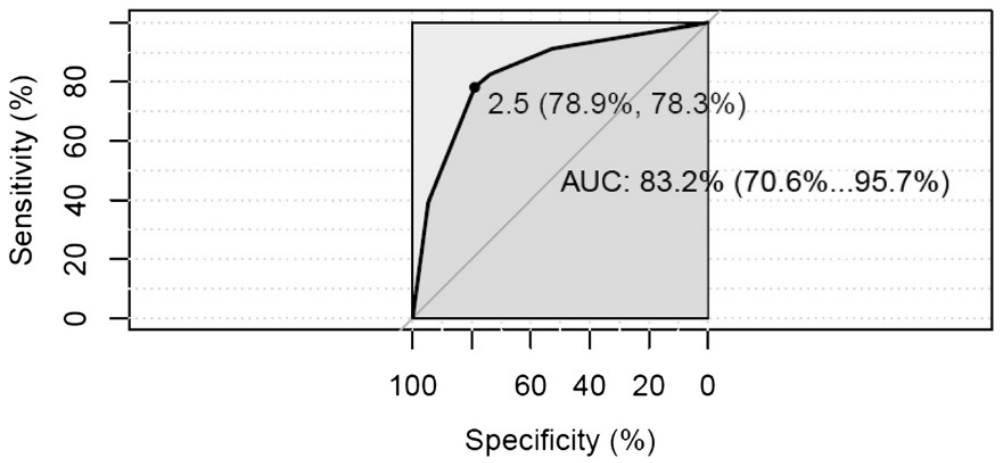

Figure 9 Receiver operating characteristic (ROC) curves. Sitting position, Rater 1 and Rater 2 with estimated area under curve AUC (95\% Cl). The optimal cut-off points for AUC are 1.5 and 2.5.

deciding whether rehabilitation for eye-head movement control impairment is necessary.

Based on the results from previous research, methodological adaptations to the tests were made in order to adapt them to a clinical environment. The measurement of quantities, such as velocity and range of motion, is impossible without technical equipment; therefore, our tests were rated on the quality of movements. In the present study, impaired quality of movement control was characterized by a short interruption during the change of direction and irregular or uncoordinated, decelerated eye movements. Decreased active stabilization of the head position during the eye movements test was also a positive rating criterion. Difficulty in keeping a fixed gaze during the gaze stability test was a regular occurrence in the patient group and was a criterion for a positive rating. Difficulty in dissociating head and eye movements in the sequential test was typical and clearly visible for a positive rating in this test.

The rating of the head-eye movement control tests used in this study can be easily learned from a short instruction video.

The methodology had to be adapted so that the tests could be videotaped. The chosen videotaping method meant that we were only able to measure horizontal eye movement. Other movement directions would have resulted in the pupil being covering by the eye-lid on the tape. This limitation would not be present in daily clinical practice, where vertical and diagonal movements could also be assessed".

To exclude the impact of pain avoidance strategies [28] and mimic reactions from the videos, an inclusion criterion was a symptom-free active range of $45^{\circ}$ head rotation to both sides.

There are certain limitations in the present study that need to be addressed. The intake of drugs was not considered. Since medication may have an influence on the velocity of head-eye movements, this information should be provided. The incidence of light was not standardized but, as there was no direct light from any side, it did not disturb the participants.

Contrary to clinical practice, raters were allowed to watch the videos several times. This may have improved inter-observer reliability. In clinical practice however, the physiotherapist is also able to repeat testing and, in addition, use symptom provocations and other assessments for clinical diagnostics. Unexpectedly, no male patients were included in the study because of recruitment 


\section{Test in standing, rater 1}

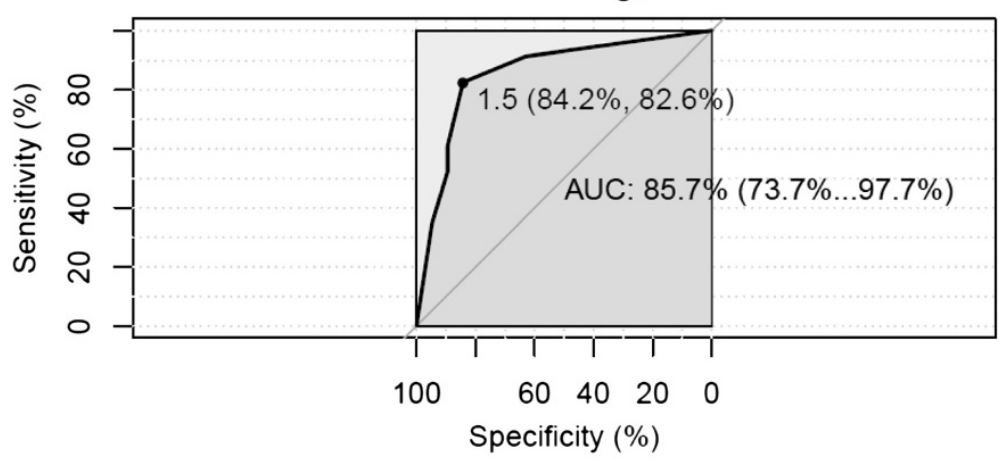

Test in standing, rater 2

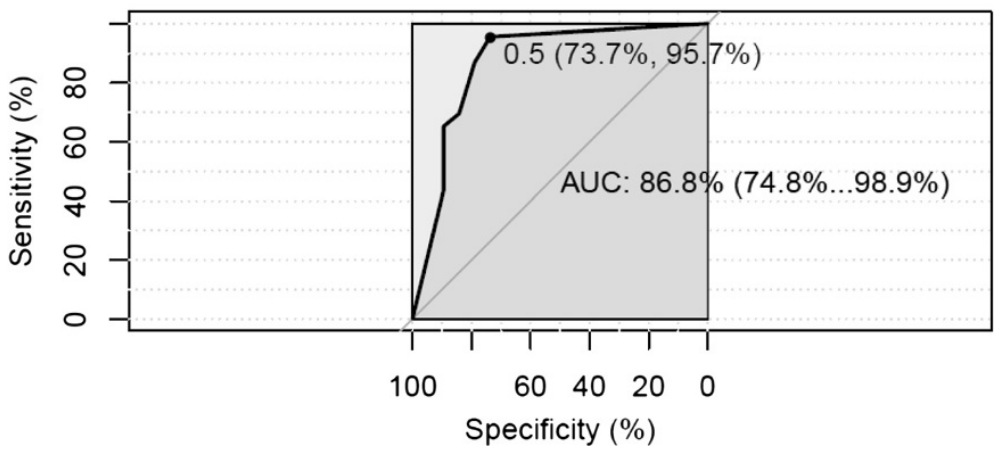

Figure 10 Receiver operating characteristic (ROC) curves. Standing position, Rater 1 and Rater 2 with estimated area under curve AUC $(95 \% \mathrm{Cl})$. The optimal cut-off points for AUC are 1.5 and 0.5 .

problems. However, neck pain and WAD are more common in women $[2,29]$.

Contrary to Treleaven and colleagues [3], the study also included patients with no neck acceleration trauma. The reason for including these subjects was that research has shown that head-eye coordination impairment is also prevalent in neck pain patients without trauma $[29,30]$. Two participants of the controls reported minor historical neck trauma but, since they had had no neck pain problems for many years, they were also included in the control group.

Further research should evaluate test-retest reliability in patients with stable symptoms. A correlation between head-eye coordination and NDI scores was not evaluated in this study, nor were differences in the performance of the trauma and non-trauma patient groups. These could be of further interest.

Table 6 Results of diagnostic odds ratio DOR by cut-off $\geq 2 / 5, \geq 3 / 5, \geq 4 / 5$ positive tests

\begin{tabular}{lccc}
\hline Rater/ Test & Cut-off $\geq \mathbf{2 / 5}$ & Cut-off $\geq \mathbf{3 / 5}$ & Cut-off $\geq \mathbf{4 / 5}$ \\
\hline Rater 1/ Test sitting & 18.6 & 15.9 & 24 \\
Rater 2/ Test sitting & 13.3 & 13.5 & 10 \\
Rater 1/ Test standing & 25.3 & 13.2 & 9.2 \\
Rater 2/ Test standing & 25.0 & 12.2 & 15.9 \\
\hline
\end{tabular}

Moreover, following diagnosis and treatment, changes in head-eye coordination tests should be evaluated in relation to changes in patients' limitations in daily life.

\section{Conclusion}

Visual assessment by physiotherapists of head-eye movement control tests is reliable. No differences between test performance in the sitting and standing positions were identified: the test battery can be reduced to five tests. Our subsequent recommendation was that tests be performed in the sitting position. The test battery can discriminate between patients with chronic neck pain and healthy controls. Two or more positive tests out of five can be interpreted as impaired head-eye movement control. Further research is needed to determine test-retest stability and responsiveness.

Table 7 Results of specificity Sp, sensitivity Sn, negative predictive value NPV, positive predictive value PPV Results by cut-off $\geq 2 / 5$

\begin{tabular}{lllll}
\hline Rater/ Test & Sp & Sn & NPV & PPV \\
\hline Rater 1/ Test sitting & 73.68 & 86.96 & 82.35 & 80.00 \\
Rater 2/ Test sitting & 73.68 & 82.61 & 77.78 & 79.17 \\
Rater 1/ Test standing & 84.21 & 82.61 & 80.00 & 86.36 \\
Rater 2/ Test standing & 78.95 & 86.96 & 83.33 & 83.33 \\
\hline
\end{tabular}




\section{Additional file}

\section{Additional file 1: Test description and instructions.}

\section{Competing interests}

The authors declare that they have no competing interests.

\section{Authors' contributions}

EDC, JAH, JK and HL designed the study. EDC and JAH collected subjects' data. AM performed statistical analysis. All authors were involved in writing the manuscript. All authors read and approved the final manuscript.

\section{Authors' information}

EDC: physiotherapist FH MAS ZHAW, PT OMT IFOMT, CRAFTA ${ }^{\oplus}$, owner of a physiotherapy practice in the -Zentrum für Manuelle Therapie- Zurich, instructor at the University of Zurich, Department of Chiropractic Medicine, CH-8008 Zurich. JAH: physiotherapist FH MAS ZHAW, PT, OMT IFOMT, owner of a physiotherapy practice in $\mathrm{CH}-8132$ Egg, instructor Kaltenborn- EvjenthConzept $^{\oplus}$. AM: physiotherapist, MSc Statistics, Institute of Physiotherapy, School of Health Professions, ZHAW Zurich University of Applied Sciences, $\mathrm{CH}-8401$ Winterthur. HL: physiotherapist, MSc, PhD, Researcher and Lecturer, Institute of Physiotherapy, School of Health Professions, ZHAW Zurich University of Applied Sciences, CH-8401 Winterthur. JK: physiotherapist, MSC, PhD, Head of Research and Development, Institute of Physiotherapy, School of Health Professions, ZHAW Zurich University of Applied Sciences, CH-8401 Winterthur.

\section{Acknowledgments}

Maja Patroncini and Susanne Hannig rated the videos. Anne Kathrin Rausch-Osthof and Karen Linwood-Williams translated the original manuscript into English. Furthermore, we would like to thank all participants in the study and our partners for their support.

\section{Author details}

${ }^{1}$ Clinic for Manual Therapy, Zeltweg 83, 8032, Zurich, Switzerland. ²Zurich University of Applied Sciences, School of Health Professions, Institute of Physiotherapy, 8400, Winterthur, Switzerland. ${ }^{3}$ Provital Physiotherapy \& GRAVITYtraining, Büelstrasse 29, 8132, Egg, Switzerland.

Received: 12 June 2013 Accepted: 7 January 2014

Published: 14 January 2014

\section{References}

1. Fejer R, Kyvik KO, Hartvigsen J: The prevalence of neck pain in the world population: a systematic critical review of the literature. Eur Spine J 2006, 15(6):834-848.

2. Haldeman S, Carroll L, Cassidy JD, Schubert J, Nygren A: The bone and joint decade 2000-2010 task force on neck pain and its associated disorders: executive summary. J Manipulative Physiol Ther 2009, 32(2 Suppl):S7-S9.

3. Treleaven J, Jull G, Grip H: Head eye co-ordination and gaze stability in subjects with persistent whiplash associated disorders. Man Ther 2011, 16(3):252-257.

4. Gimse R, Bjørgen IA, Straume A: Driving skills after whiplash. Scand J Psychol 1997, 38(3):165-170.

5. Gimse R, Tjell C, Bjørgen IA, Saunte C: Disturbed eye movements after whiplash due to injuries to the posture control system. J Clin Exp Neuropsychol 1996, 18(2):178-186.

6. Hildingsson C, Wenngren BI, Bring G, Toolanen G: Oculomotor problems after cervical spine injury. Acta Orthop Scand 1989, 60(5):513-516.

7. Hildingsson $\mathrm{C}$, Wenngren $\mathrm{Bl}$, Toolanen $\mathrm{G}$ : Eye motility dysfunction after soft-tissue injury of the cervical spine. A controlled, prospective study of 38 patients. Acta Orthop Scand 1993, 64(2):129-132.

8. Wenngren Bl, Pettersson K, Lowenhielm G, Hildingsson C: Eye motility and auditory brainstem response dysfunction after whiplash injury. Acto Otolaryngol 2002, 122(3):276-283.

9. Yahia A: Chronic neck pain and vertigo: is a true balance disorder present? Ann Phys Rehabil Med 2009, 52(7-8):556-567.
10. Humphreys $K$, Irgens $P$ : The effect of a rehabilitation exercise program on head repositioning accuracy and reported levels of pain in chronic neck pain subjects. J Whiplash Relat Disord 2002, 1:99-112.

11. Heikkila H, Wenngren B: Cervicocephalic kinesthetic sensibility, active range of cervical motion, and oculomotor function in patients with whiplash injury. Arch Phys Med Rehabil 1998, 79(9):1089-1094.

12. Revel M, Andre-Deshays C, Minguet M: Cervicocephalic kinesthetic sensibility in patients with cervical pain. Arch Phys Med Rehabil 1991, 72(5):288-291.

13. Peterson BW: Current approaches and future directions to understanding control of head movement. Prog Brain Res 2004, 143:369-381.

14. Armstrong B, McNair P, Taylor D: Head and neck position sense. Sports Med 2008, 38(2):101-117.

15. Kristjansson E, Oddsdottir GL: "The Fly": a new clinical assessment and treatment method for deficits of movement control in the cervical spine: reliability and validity. Spine (Phila Pa 1976) 2010, 35(23):E1298-E1305.

16. Tjell C, Rosenhall U: Smooth pursuit neck torsion test: a specific test for cervical dizziness. Am J Otol 1998, 19(1):76-81.

17. Grip H, Jull G, Treleaven J: Head eye co-ordination using simultaneous measurement of eye in head and head in space movements: potential for use in subjects with a whiplash injury. J Clin Monit Comput 2009, 23(1):31-40

18. Boyling JD, Jull G: Grieve's Modern Manual Therapy, The Vertebral Column. Thirdth edition. Edinburgh: Elsevier Churchill Livingstone; 2004.

19. Jull G, Sterling M, Falla D, Treleaven J, O'Leary S: Whiplash, Headache, and Neckpain. London: Elsevier Churchill Livingston; 2008.

20. Treleaven J: Sensorimotor disturbances in neck disorders affecting postural stability, head and eye movement control. Man Ther 2008, 13(1):2-11.

21. Luomajoki $\mathrm{H}, \mathrm{Kool} J$, de Bruin ED, Airaksinen O: Reliability of movement control tests in the lumbar spine. BMC Musculoskelet Disord 2007, 8:90.

22. Vernon H, Mior S: The neck disability index: a study of reliability and validity. J Manipulative Physiol Ther 1991, 14(7):409-415.

23. Vernon H: The neck disability index: state-of-the-art, 1991-2008. J Manipulative Physiol Ther 2008, 31(7):491-502.

24. Swanenburg J, Humphreys K, Langenfeld A, Brunner F, Wirth B: Validity and reliability of a German version of the neck disability index (NDI-G). Man Ther 2013. in press

25. Teng CC, Chai H, Lai DM, Wang SF: Cervicocephalic kinesthetic sensibility in young and middle-aged adults with or without a history of mild neck pain. Man Ther 2007, 12(1):22-28.

26. Cinelli M, Patla A, Stuart B: Age-related differences during a gaze reorientation task while standing or walking on a treadmill. Exp Brain Res 2008, 185(1):157-164.

27. Landis JR, Koch GG: The measurement of observer agreement for categorical data. Biometrics 1977, 33(1):159-174.

28. Sjölander P, Michaelson P, Jaric S, Djupsjöbacka M: Sensorimotor disturbances in chronic neck pain-range of motion, peak velocity, smoothness of movement, and repositioning acuity. Man Ther 2008, 13(2):122-131.

29. Humphreys BK: Cervical outcome measures: testing for postural stability and balance. J Manipulative Physiol Ther 2008, 31(7):540-546.

30. Treleaven J, Clamaron-Cheers C, Jull G: Does the region of pain influence the presence of sensorimotor disturbances in neck pain disorders? Man Ther 2011, 16(6):636-640.

\section{doi:10.1186/1471-2474-15-16}

Cite this article as: Della Casa et al:: Head-Eye movement control tests in patients with chronic neck pain; Inter-observer reliability and discriminative validity. BMC Musculoskeletal Disorders 2014 15:16. 